\title{
Monitoreo ambulatorio y generación de alertas tempranas ante anomalías cardiacas usando dispositivos móviles
}

\author{
José Ignacio Arias Contreras", Sandra Esperanza Nope Rodríguez, Eduardo Caicedo Bravo \\ Universidad del Valle. Santiago de Cali, Colombia
}

Recibido 23 de marzo de 2016. Aceptado 9 de febrero de 2017

\begin{abstract}
Resumen-En este artículo se presenta un sistema portátil para el monitoreo ambulatorio del ritmo cardiaco y la detección temprana de las arritmias cardiacas de mayor riesgo. El sistema consta de un sensor con tres electrodos superficiales para la captura de la señal ECG, la cual se transmite vía Bluetooth a un dispositivo móvil con Android OS, en donde se realiza el análisis de la señal capturada durante lapsos de $5 \mathrm{~s}$. El sistema propuesto distingue entre Ritmo Normal (Ritmo Sinusal - RS), Taquicardia Ventricular $(T V)$, Fibrilación Ventricular $(F V)$ y Asistolia, con una precisión del 100\%, 55\%, 75\% y 90\% respectivamente. Sin embargo, el sistema puede recuperarse de los errores rápidamente en el análisis de la trama subsecuente. Este trabajo se centra en el uso de dispositivos móviles de uso cotidiano, multitarea y de fácil acceso, implementando algoritmos en el dominio del tiempo para la extracción de parámetros, los cuales son idóneos para ser usados en aplicaciones móviles principalmente por su baja carga computacional y posibilidad de ejecución en tiempo real, permitiendo la detección de anomalías cardiacas de forma automática y rápida sin la necesidad de una supervisión constante por parte de un especialista para el análisis preliminar.
\end{abstract}

Palabras Clave_Detección de arritmias, Dispositivos móviles, Monitoreo ambulatorio, Procesamiento de señales ECG.

\section{Ambulatory Monitoring and Early Warning Generation of Cardiac Anomalies Using Mobile Devices}

\footnotetext{
Abstract - This paper presents a portable system for ambulatory heart rate monitoring and early detection of cardiac arrhythmias at high risk. The system consists of a sensor with three surface electrodes to capture the ECG signal, which is transmitted via Bluetooth to a mobile device with Android OS, where the analysis is performed of the acquired signal during a time of $5 \mathrm{~s}$. The proposed system distinguishes between Normal Rhythm (Rhythm Sinus - RS), Ventricular Tachycardia (VT), Ventricular
}

\% Dirección para correspondencia: jose.arias.contreras@correounivalle.edu.co DOI: https://doi.org/10.24050/19099762.n23.2018.900 
Fibrillation $(V F)$ and Asystole with an accuracy of $100 \%, 55 \%, 75 \%$ and $90 \%$ respectively. However, the system can quickly recover from errors in the subsequent analysis frame. This work focuses on using regular mobile devices which have multitasking and easy access characteristics, implementing algorithms in time domain for extracting parameters that are suitable to use in mobile applications, mainly because of their low computational load and possibility of execution in real time, allowing the detection of cardiac abnormalities automatically and quickly without the need of constant supervision by a specialist for preliminary analysis.

Keywords - Detection of arrhythmias, Mobile devices, Ambulatory monitoring, ECG signal processing.

\title{
MONITORAMENTO AMBULATORIAL E GERAÇÃO DE AVISO PRÉVIO DE ANOMALIAS CARDÍACAS USANDO DISPOSITIVOS MÓVEIS
}

\begin{abstract}
Resumo - Neste artigo se apresenta um sistema portátil para o monitoramento da freqüência cardíaca ambulatorial e detecção precoce das arritmias cardíacas de mais risco. O sistema possui um sensor com três eletrodos superficiais para pegar o sinal ECG, o qual é transmitido via Bluetooth para um dispositivo móvel com Android OS, onde se faz a análise do sinal capturado durante um período de 5 s. O sistema proposto distingue entre Normal Ritmo (Ritmo Sinusal - RS), Taquicardia Ventricular (TV), Fibrilação Ventricular (FV) e Assistolia, com uma precisão do 100\%, 55\%, 75\% e 90\%, respectivamente. Porém, o sistema pode - se recuperar rapidamente dos erros na análise do quadro subsequente. Este trabalho centra-se no uso de dispositivos móveis de utilização diária, multitarefa e utilização acessível, implementação de algoritmos no domínio do tempo para a extração de parâmetros que são adequados para utilização em aplicações móveis, principalmente pela baixa carga computacional e possibilidade de execução em tempo real, permitindo a detecção de anormalidades cardíacas numa forma automática e rápida sem a necessidade de um controlo constante por um especialista para análise preliminar.
\end{abstract}

Palavras-chave—Detecção de arritmias, Dispositivos móveis, Monitorização ambulatorial, Processamento de sinal ECG.

\section{INTRODUCCIÓN}

$\mathrm{S}$ egún la Organización Mundial de la Salud (OMS), las Enfermedades Cardiovasculares (ECV), son la principal causa de muerte a nivel mundial. Un estimado de 17,7 millones de personas murieron a causa de ECV en 2015 , que representa un $31 \%$ todas las muertes a nivel mundial [Web 1]. En Colombia, se constituyó en la primera causa de muerte en el 2016 debido a sus complicaciones [Web 2]. Ante una arritmia cardiaca, es fundamental reducir el tiempo de detección y atención, de forma que se minimice la posibilidad de sufrir daños cerebrales, caer en estado de coma, o, en el peor de los escenarios, la muerte.

Gran parte de la tecnología relacionada con la salud está concentrada en los centros de salud, debido principalmente a los altos costos de adquisición y mantenimiento de dicha tecnología, así como su complejidad de manejo y necesidad de tener un conocimiento especializado para interpretar adecuadamente la información capturada. Dentro de la gama de dispositivos de uso ambulatorio para el registro de la actividad eléctrica del corazón se encuentra el "Holter" [Web 3, 1], que suele utilizarse durante un periodo entre 12 a 24 horas, y corresponde a un conjunto de electrodos superficiales (entre 3 a 10 dependiendo del fabricante) para almacenar las señales ECG, pero el análisis de la señal es realizada posteriormente por un especialista. Sistemas comerciales como: "Pulse-ring" [Web 8], "Mio
Alpha" [Web 6], o el "Beurer pm-100" [Web 7], permiten el análisis básico de las señales ECG, donde sólo le permite conocer al usuario la frecuencia cardiaca instantánea.

El desarrollo constante en el campo de los dispositivos móviles (PDA - Personal Digital Assistant) en cuanto a reducción de tamaño, portabilidad, poder de procesamiento, variedad de interconexión y accesibilidad (menor costo y mayor distribución); los ha convertido en dispositivos de uso cotidiano para la gran mayoría de personas, con alto potencial para desarrollar aplicaciones no sólo en el campo del entretenimiento y las comunicaciones, sino también en el campo de la salud. De este modo, es posible facilitar el monitoreo y análisis de bio-señales haciendo uso de la capacidad de procesamiento de los dispositivos móviles actuales, que, en el caso de las arritmias cardiacas, garantizarían una reducción en el tiempo de detección y atención al paciente.

Existen sistemas portables de captura, registro y envío a través de internet de señales ECG a un centro de salud o especialista, como el "AliveCor Heart Monitor" [Web 4] [2, 3]. Este tipo de sistemas tiene el inconveniente que durante el envío de la información ésta se puede alterar o perder en el canal de comunicación, y es susceptible a la disponibilidad de una red para el acceso a internet, así como la experticia y disponibilidad del especialista para el análisis. Así mismo, por lo general requieren de dispositivos adicionales, por ejemplo, [Web 4] requiere de un accesorio que se encaja a presión en la parte posterior del Smartphone 
en donde el usuario debe presionar los dedos sobre dos contactos metálicos que hacen las veces de electrodos, para que la señal ECG pueda ser capturada; esto implica que el usuario no pueda realizar actividades que requieran el uso de sus dos manos, y por tanto, dificulta la continuidad en el registro de la señal. En [2] además de la aplicación móvil, es necesario adquirir el sensor comercial "Kardia" que consta de tres electrodos superficiales para la lectura de la señal ECG que se transmite vía Bluetooth al dispositivo móvil y de allí a un centro de salud. En [3] se utilizan tres electrodos superficiales y un sensor de temperatura. En todos los casos anteriores, los dispositivos permiten sólo la captura, visualización y registro de la señales, y no cuentan con la capacidad de analizar la señal ECG.

En $[4,5,6]$ se desarrollaron aplicaciones que son capaces de extraer sólo la información de frecuencia cardiaca a partir de la señal ECG. Con base en esta información, en [4] pueden determinar la presencia de taquicardia o bradicardia y generar una alerta. En $[5,6]$ se desarrollaron aplicaciones que utilizan el sensor comercial "Zephyr HXM" el cual tiene la capacidad de calcular la frecuencia cardiaca y enviarla al dispositivo móvil; ambas aplicaciones establecen rangos de normalidad y anormalidad (bradicardia o taquicardia) de la frecuencia cardiaca para generar alertas junto con la ubicación geográfica del usuario.

En el apartado II inicia con la descripción general del sistema y posteriormente se detallan las etapas y algoritmos usados para el procesamiento de la señal ECG en el dispositivo móvil. El apartado III se presenta los resultados obtenidos y la metodología usada para obtenerlos. Finalmente, en el apartado IV se exponen las principales conclusiones y se plantea el trabajo futuro.

\section{Materiales y Métodos}

La Fig. 1 muestra el diagrama de bloques general del sistema de identificación del ritmo cardiaco que está compuesto por tres bloques: "Captura de la Señal ECG", "Preprocesamiento" e "Identificación del Ritmo Cardiaco".
La identificación del ritmo cardiaco se realiza en cascada, primero se detecta la presencia o no de Asistolia, sino corresponde a Asistolia se verifica si se trata o no de un Ritmo Normal, finalmente, el sistema identifica si se trata de una Taquicardia Ventricular (TV) o de una Fibrilación Ventricular $(F V)$. A continuación se describe cada uno de los bloques que componen el sistema desarrollado:

\subsection{Bloque 1: Captura de la señal ECG}

Antes de iniciar el procesamiento, es necesario emparejar el circuito sensor con el dispositivo móvil, para ello: 1) se debe encender el sensor y el Bluetooth del dispositivo móvil, 2) se verifica que el dispositivo móvil esté visible al aparecer con el nombre "FireFly-C584" dentro del listado que se genera con la opción "Buscar Dispositivos", 3) Al elegir el "FireFly-C584" haciendo clic sobre él, se introduce inicialmente la contraseña "1234" para que se realice el emparejamiento, y 4) abrir la aplicación, ingresar el nombre del sensor (FireFly-C584) y presionar el botón "Conectar con:" para que inicie la transmisión de datos de forma continua desde el sensor hacia el disposi-tivo móvil. La señal capturada es amplificada (980 veces), digitaliza (12 bits a $313 \mathrm{~Hz}$ ) y enviada al dispositivo móvil. La captura de las señales ECG se realiza mediante tres electrodos superficiales ubicados sobre el área del corazón en una configuración como la que se presenta en la Fig. 2, el sensor amplifica la señal, la digitaliza y envía mediante un módulo de comunicación Bluetooth al dispositivo móvil. Ya en el dispositivo móvil la señal es segmentada en tramas de $5 \mathrm{~s}$ (1.565 muestras). El análisis de segmentos de unos pocos segundos ( 3 a $10 \mathrm{~s}$ ) de duración ha demostrado tener buenos resultados $[7,8,11]$, en este caso los segmentos evaluados tienen una duración de $5 \mathrm{~s}$ lo que garantiza que haya la suficiente información para extraer las características que permiten identificar los diferente ritmos cardiacos, y al mismo tiempo, es un lapso que introduce un retraso mínimo en la generación de alarmas.

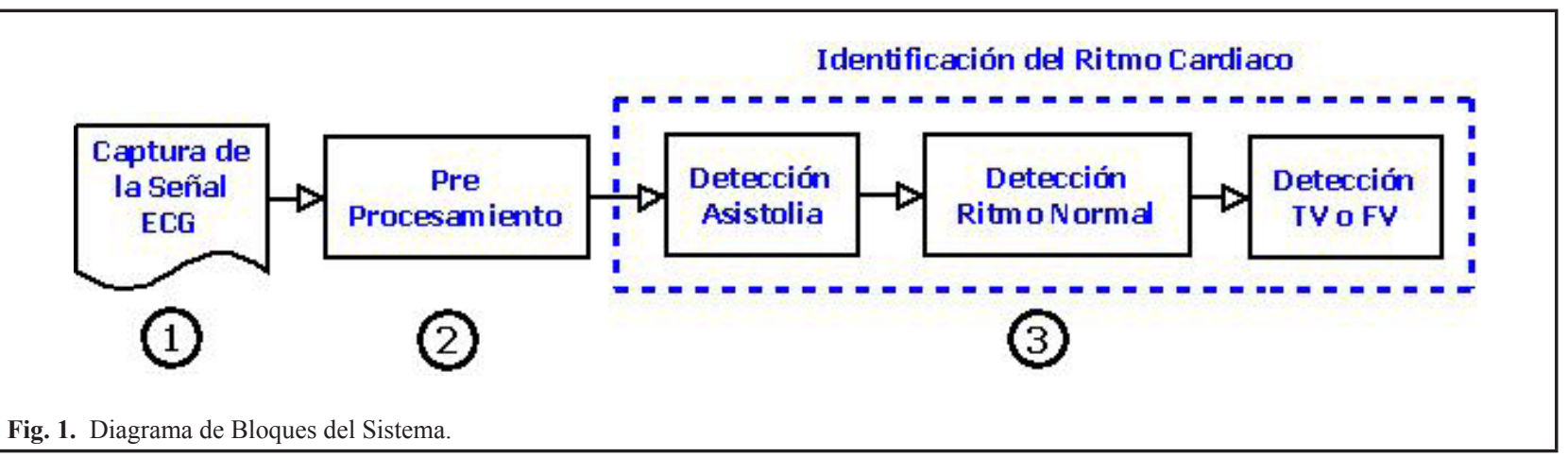




\subsection{Bloque 2: Pre-procesamiento}

Las señales ECG son sensibles al ruido e interferencias debido a su baja magnitud del orden de los $\mathrm{mV}$, cuyo efecto puede minimizarse mediante el uso de un filtro pasa-banda $[7,11,16,20,21]$ que, elimina componentes de baja frecuencia como los generados por la respiración, movimientos del paciente y el movimiento de los electrodos (cambio de la impedancia electrodo-piel) las cuales suelen estar por debajo de los $0.5 \mathrm{~Hz}$, de no realizarse este filtrado, se presenta un desplazamiento de la línea de base (drift baseline o wander baseline) [24], línea sobre la cual se montan las ondas generadas durante la actividad eléctrica del corazón. También es deseable suprimir interferencias como los introducidos por el sistema de medida, la interferencia de la red eléctrica que corresponde a los $60 \mathrm{~Hz}$ y la actividad eléctrica de los músculos (Señales Electromiográficas - EMG) en el rango de los 50 a $150 \mathrm{~Hz}$.

Se implementó en la aplicación un filtro IIR de tipo Butterworth que, al tener un orden considerablemente menor a un filtro FIR, sugiere un menor costo computacional $[7,11,20]$. Se diseñó para que la banda de paso sea de 1 a $30 \mathrm{~Hz}$, rango que incorpora el espectro de frecuencia de los ritmos de interés $[8,11]$. De este modo, la función de transferencia que describe el filtro pasa-banda utilizado corresponde a la descrita en (1):

$$
h(s)=\frac{0,0029 s^{8}-0,0116 s^{6}+0,0174 s^{4}-0,0116 s^{2}+0,0029}{s^{8}-6,469 s^{7}+18,445 s^{6}-30,323 s^{5}+31,469 s^{4}-21,124 s^{3}+8,958 s^{2}-2,193 s+0,237}
$$

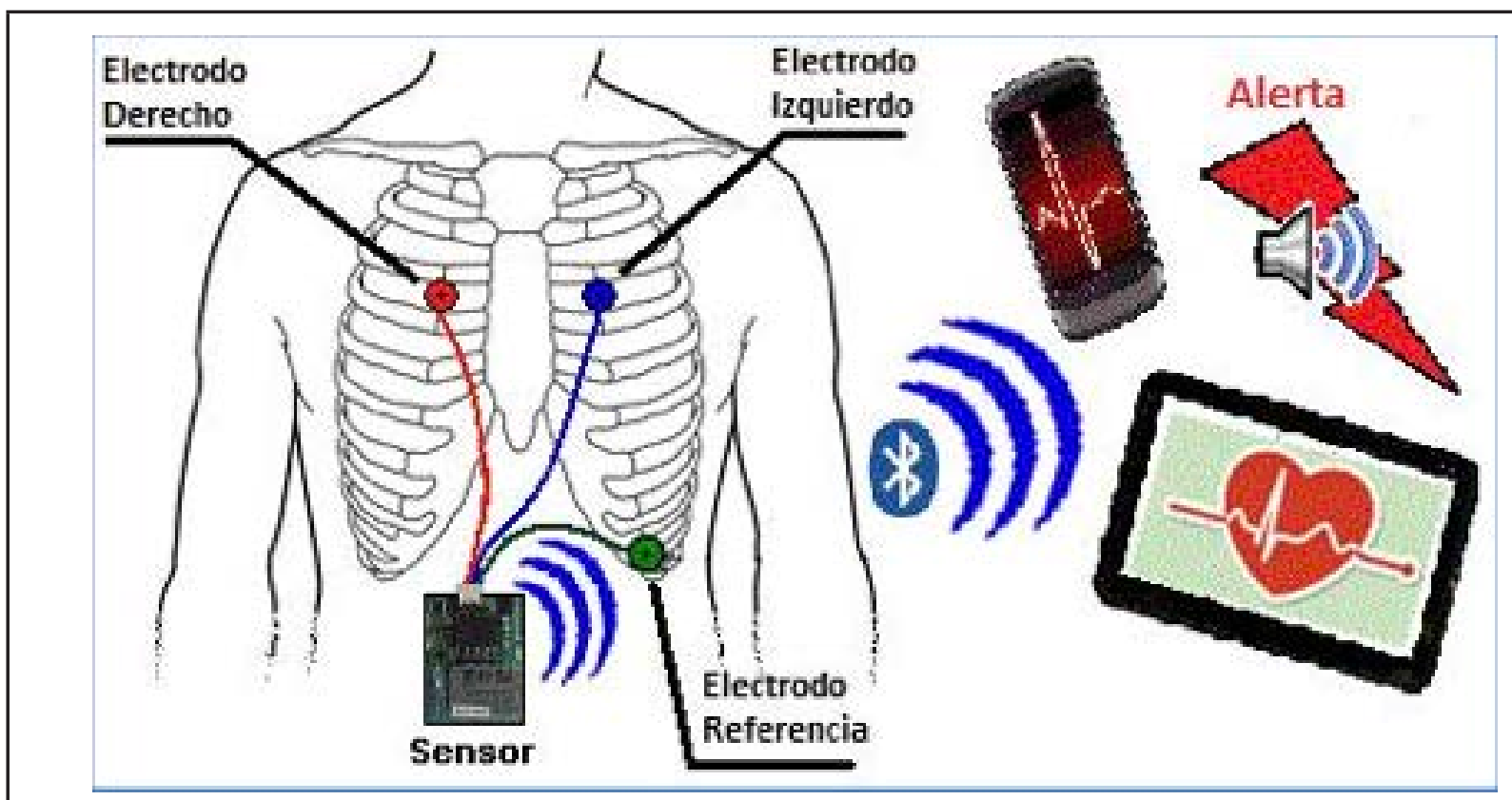

Fig. 2. Imagen general del sistema

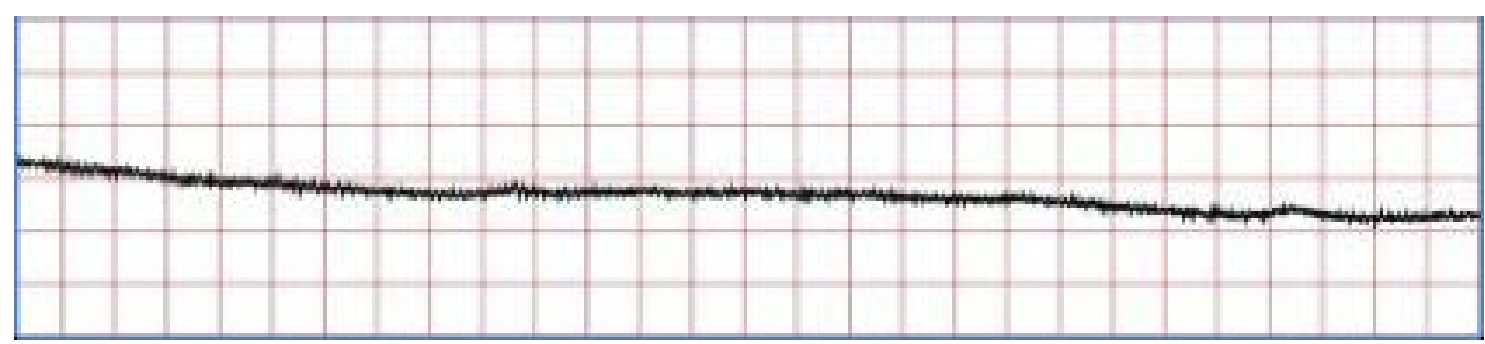

Fig. 3. Ritmo Asistolia. 


\subsection{Bloque 3: Identificación de Ritmo Cardiaco}

En este bloque se buscó el desarrollo de algoritmos de baja carga computacional, teniendo en cuenta que los dispositivos móviles no son dispositivos que estén dedicados únicamente al análisis de señales ECG, y por el contrario, puede ejecutar una gran variedad de tareas simultáneamente como: reproducción multimedia, navegación Web, comunicación, etc.

Una opción interesante son los algoritmo usados por los Desfibriladores Externos Automáticos (DEA) o Implantables $(D A I)[7,13]$ para la detección de las Arritmias Ventriculares $(A V)$ como la FV y TV [9] con el objeto de revertir dichas arritmias a través de descargas eléctricas [Web 5]. Estos dispositivos estiman:

a. La "Frecuencia Ventricular" y con base en ella, pueden detectar Taquiarritmias Ventriculares con una sensibilidad (Normal o Anormal) cercana al $100 \%$, pero una especificidad (discriminación entre anomalías) baja, por lo cual posteriormente se agregaron más parámetros como los mencionados en b, c y d para aumentar la especificidad.

b. La "Estabilidad de la Frecuencia Cardiaca" permite distinguir entre TV Monomórficas, TV Polimórficas o Fibrilación Auricular (FA) logrando una sensibilidad del $100 \%$ y una especificidad del $81,8 \%$ [9].

c. El "Inicio Súbito (Sudden Onset)" permite diferenciar entre una Taquicardia Sinusal (asociada al aumento gradual de la frecuencia provocado posiblemente por ejercicio físico) de una TV cuyo inicio suele ser mucho más repentino.

d. La "Morfología o Anchura" permite discriminar entre arritmias supraventriculares (no letales) de las ventriculares FV y TV.

De las características de la señal se consideraron los siguientes dominios para la detección de anomalías: el Tiempo, la Frecuencia, Tiempo-Frecuencia y Complejo [7, $11,12]$. Entre las características extraídas en el domino del tiempo, suelen utilizarse: "La Función de Autocorrelación (autocorrelation function - $A C F$ )" [8], "Intervalo de Cruce de Umbrales (Threshold Crossing Interval - TCI)" [8, 14], "Función de Densidad de Probabilidad (Probability Density Function - PDF)" y "El Potencial Isoeléctrico $(P I)$ " [11, 15]. El "Algoritmo de Pan-Tompkins" [16, 12, 17, 18], la "Medida de Complejidad", el "Chequeo Secuencial de la Hipótesis" [7], "La Estimación del tiempo que la señal permanece por encima o por debajo de un umbral definido" [15], "Cálculo de los Tramos RR" (distancia entre picos $R$ ) [19]. Para el análisis en el domino de la frecuencia de la señal ECG usualmente se realiza mediante la evaluación del espectro de potencia de la señal, en este dominio se encuentran: "Transformada Rápida de Fourier (Fast
Fourier Transform - FFT)", "Análisis de la Distribución de Amplitud Modificada (Modified Amplitude Distribution Analysis - MADA)", "Análisis del Espectro de Singularidad Multifractal (Multifractal Singularity Spectrum Analysis)" [8]. Los algoritmos basados en las transformaciones Tiempo-Frecuencia [7, 11] son: "La Transformada de Fourier de Tiempo Corto (Short-time Fourier transform - STFT)" y "La Transformada Wavelet". Finalmente, dado que las arritmias ventriculares son consideradas procesos fisiológicos no lineales de diversa complejidad, pueden ser cuantificados usando técnicas como la "Medida de Complejidad de Lempel y Ziv", que fue usada para estimar la complejidad de las arritmias ventriculares en [11, 18].

Como se mencionó anteriormente, es importante implementar estrategias de reconocimiento de bajo nivel de procesamiento para garantizar la detección en tiempo real, y por el hecho de ser usado por un hardware que no es dedicado sólo a la tarea de monitoreo del ritmo cardiaco, sino que por el contrario, debe compartir recursos con otras aplicaciones. Por lo anterior, se descartaron algoritmos que requieran procesamiento adicional para la transformación de la señal a otro dominio, y se concentró el análisis en características en el dominio del tiempo. Sin embargo, se integró más de una característica en el dominio temporal, dado los resultados alcanzados por $[7,8,12,15,18]$ que sugieren la potencialidad de mejorar la capacidad de discriminación de las diferentes anomalías si se integra más de un parámetro en este dominio [15]. La forma como estos algoritmos son implementados se describe a continuación, y se integraron para trabajar en cascada, de modo que no deben calcularse simultáneamente, sino que la necesidad de estimar otra característica depende del ritmo cardiaco que se vaya descartando. La primera arritmia que se detecta es la presencia o no de Asistolia, en caso de no verificarse una asistolia, se verifica si se trata de un Ritmo Sinusal (RS), de no ser este el caso, el sistema finalmente determina si se trata de un episodio de Taquicardia Ventricular (TV) o de una Fibrilación Ventricular (FV).

\subsubsection{Detección de Asistolia}

El ritmo de Asistolia Fig. 3, se caracteriza por la falta de actividad eléctrica y mecánica del corazón (sin pulso), por lo tanto, la señal ECG es de baja potencia ya que la actividad eléctrica se concentra alrededor de la línea de base. Existen técnicas muy simples como la definición de umbrales [22], así, si la amplitud de la señal está por debajo de $80 \mu \mathrm{V}$ se trata de una Asistolia. Una mejor opción es realizar la inferencia a partir del cálculo de la potencia del segmento [11] y compararla con un umbral preestablecido.

Esta última idea fue la implementada en el sistema propuesto, para ello, se dividió el segmento ECG de $5 \mathrm{~s}$ en dos partes iguales (sub-segmentos), y a cada una se le 
calcula la potencia haciendo uso de (2). De este modo, P1 será la Potencia del Sub-segmento 1 y P2 la Potencia del Sub-segmento 2:

$$
P_{i}=10^{3} * \frac{1}{\frac{N}{2}} * \sum_{(i-1) * \frac{N}{2}}^{i * \frac{N}{2}-1} x^{\prime 2}(n) \quad i=1,2
$$

Donde i tiene el valor de uno para la sub-trama 1 y el valor de dos para la sub-trama $2, \mathrm{~N}$ es el número de muestras de la trama (1.565 muestras) que corresponde a $5 \mathrm{~s}$, y x' corresponde a los valores de la señal ECG de la trama evaluada.

Para determinar la presencia o no de Asistolia, se parte de la potencia más grande entre P1 y P2 y se compara con un umbral predefinido, al igual que los otros algoritmos de detección de Asistolia. A partir de la pruebas se encontró que un buen umbral corresponde a 20.000; Si la potencia está por debajo de dicho valor, la trama ECG evaluada será clasificado como Asistolia, en cuyo caso la aplicación genera una alarma sonora y en la pantalla se muestra un mensaje indicando que se detectó un ritmo de "Asistolia" e iniciara el análisis de una nueva trama de $5 \mathrm{~s}$. En caso contrario, la trama es analizada por la siguiente etapa "Detección de Ritmo Sinusal".

Es importante mencionar que en el desempeño de este sub-bloque influye la longitud la trama evaluada (suficientemente larga), pues si es muy corto ritmos con frecuencias cardiacas muy bajas pueden ser clasificados erróneamente con una Asistolia, por ejemplo durante una Bradicardia que se caracteriza por tener un ritmo cardiaco muy lento alrededor de los $40 \mathrm{lpm}$ (latidos por minuto) con periodos de baja actividad eléctrica de larga duración [11], similares a los presentados durante una Asistolia. La Bradicardia no es un ritmo de alto riesgo en comparación con la Asistolia, FV o TV, pero también requiere de atención ya que puede generar angina, disminución de la capacidad para la actividad física, desmayo, insuficiencia cardiaca [5].

\subsubsection{Detección Ritmo Sinusal}

Este sub-bloque "Detección de Ritmo Normal", una vez descartada la Asistolia, busca identificar si se trata de un Ritmo Normal o no. Al igual que en el caso anterior, si hay una identificación positiva de Ritmo Normal el algoritmo procede a analizar una nueva muestra de $5 \mathrm{~s}$, de lo contrario, busca identificar a través del siguiente subbloque entre Ritmos Letales como la TV/FV.

La Identificación del Ritmo Normal se realiza mediante el cálculo del "Porcentaje de Muestras en el Rango Dinámico $(P M R D)$ " descrito en [7]. Este algoritmo se fundamenta en que el Ritmo Sinusal $(R S)$ o Ritmo Normal presenta una línea base sobre la cual están superpuestas las ondas $\mathrm{P}$, el complejo QRS, la onda $\mathrm{T}$ y los segmentos isoeléctricos (segmentos sin actividad eléctrica), de forma independiente a la frecuencia cardiaca. En contraposición durante los ritmos de TV o FV no se generan a partir de una línea de base y la amplitud de la señal tiene un comportamiento oscilatorio continuo sin presentar segmentos isoeléctricos. En la Fig. 4 hay un ejemplo donde se aprecia las ondas P, QRS y T para el Ritmo Normal y la falta de estas en una Taquicardia Ventricular.

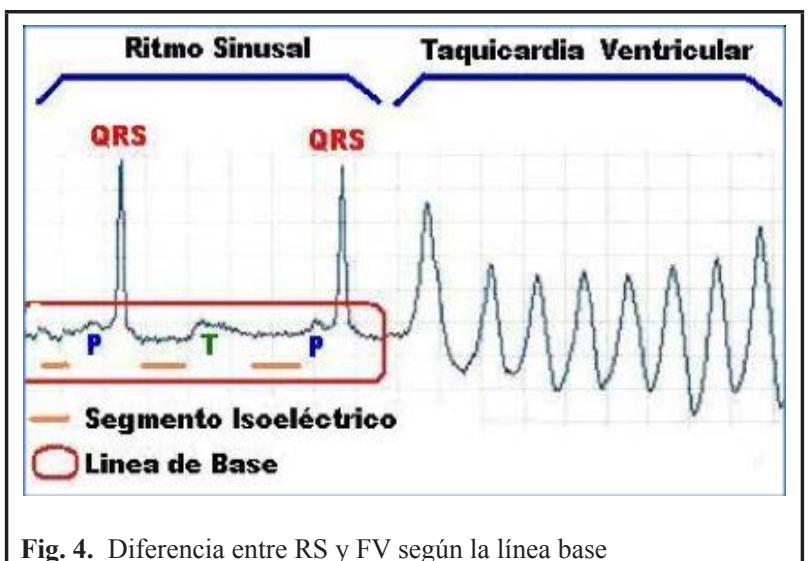

El algoritmo PMRD empieza hallando la muestra con la mayor amplitud dentro de la trama ECG evaluada, este valor se establece como el umbral superior bajo el nombre de Valor Pico Positivo (VPP), en base a este valor, se establece el umbral inferior el cual está definido como el $10 \%$ del VPP, estos dos umbrales dan lugar a un rango de valores de amplitud. Mediante el cálculo del porcentaje de muestras dentro del rango de amplitud previamente calculado, se determina si el ritmo evaluado corresponde a uno de estas dos opciones; ritmo con variaciones de baja amplitud y con segmentos isoeléctricos como los presentados en un ritmo Sinusal (RS) o en caso contrario corresponde a un ritmo como grandes variaciones de amplitud y sin segmentos isoeléctricos como es el caso de TV y FV. Así, el parámetro PMRD corresponde al porcentaje de muestras dentro de dicho rango, si este porcentaje es menor a $30 \%$ la trama evaluada corresponde a un ritmo cardiaco Sinusal, en caso contrario corresponde a un ritmo de TV o FV. En la Fig. 5 se presenta ejemplos del cálculo del parámetro PMRD para tres tramas ECG de la misma longitud, en donde A es un Ritmo Sinusal con un PMRD=11,5\% el cual es menor al 30\%, B es una Fibrilación Ventricular con $\mathrm{PMRD}=39,1 \%$, y $\mathrm{C}$ es una Taquicardia Ventricular con $\mathrm{PMRD}=44 \%$, y como se observa en estos dos últimos casos es mayor al $30 \%$. 


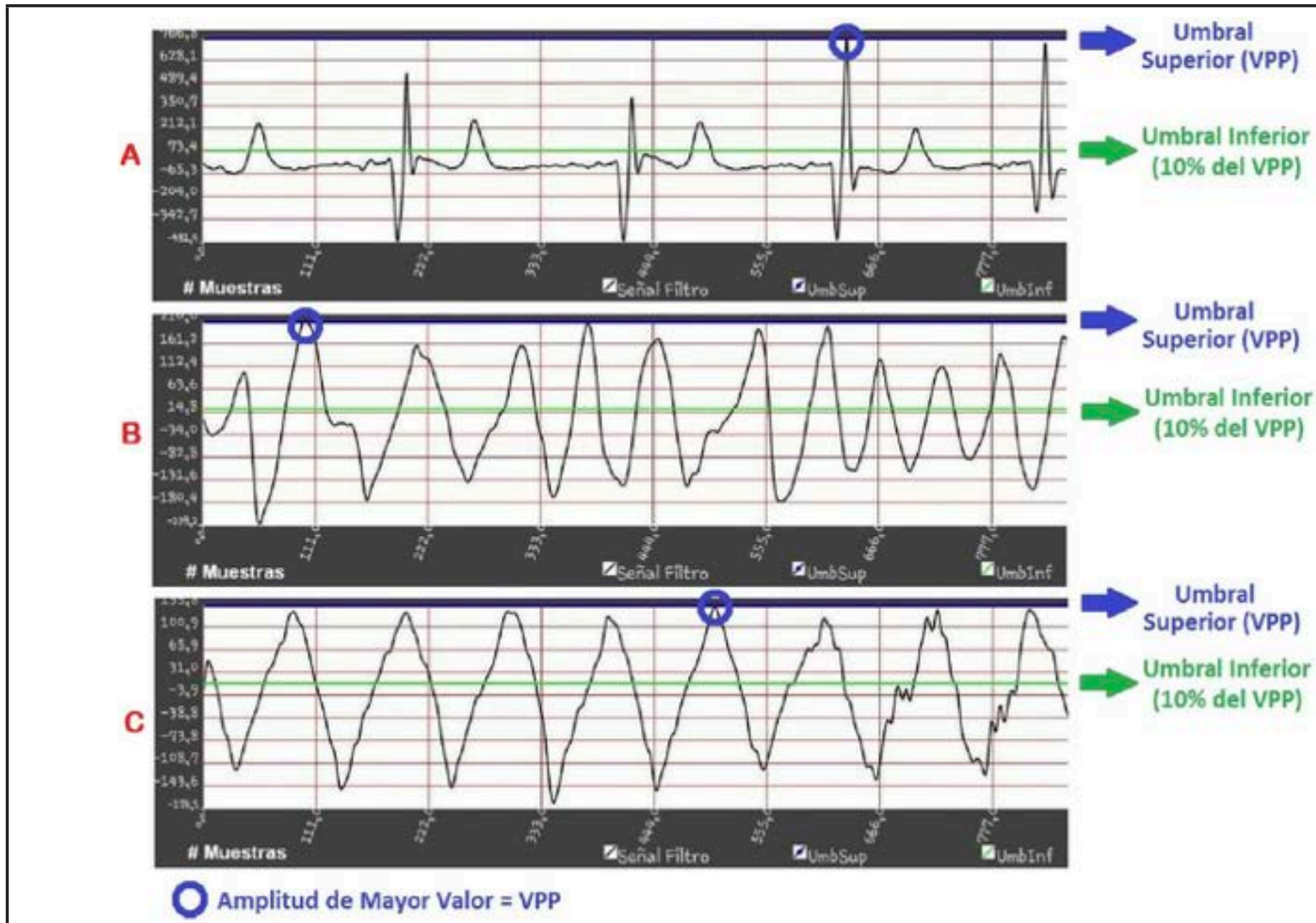

Fig. 5. Porcentaje de muestras en el intervalo VPP-10\%VPP para una señal RS (A), FV (B) y una TV (C)

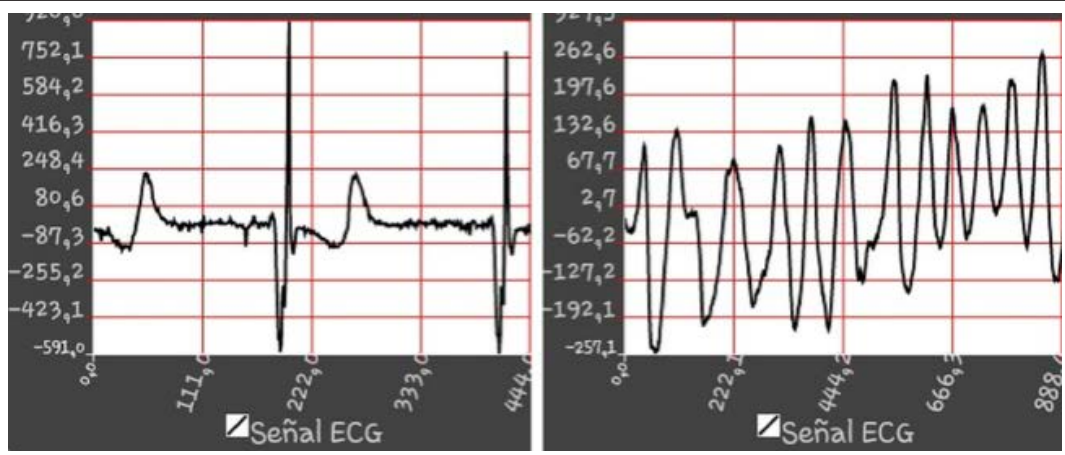

Fig. 6. Diferencia entre Picos Sanos (presentes en RS) y no Sanos (presentes en TV o FV)

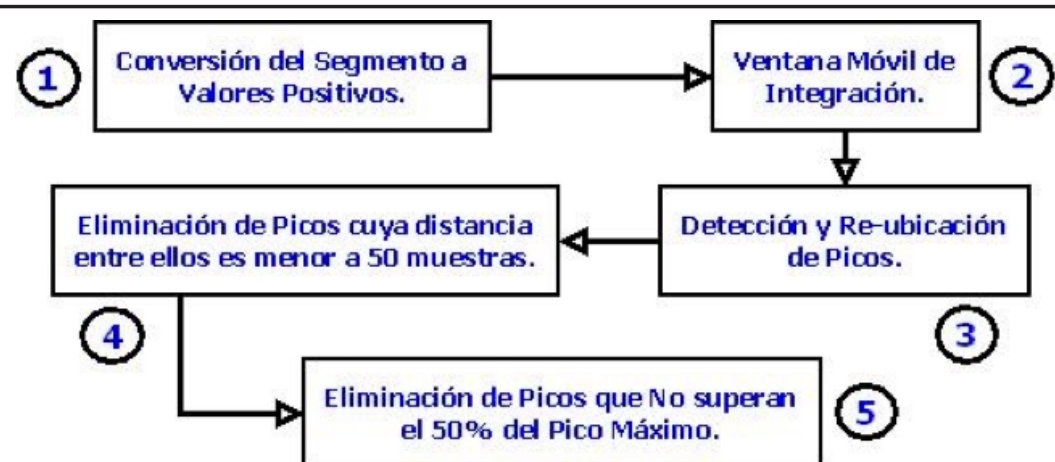

Fig. 7. Diagrama de bloques Algoritmo Detección de Picos 

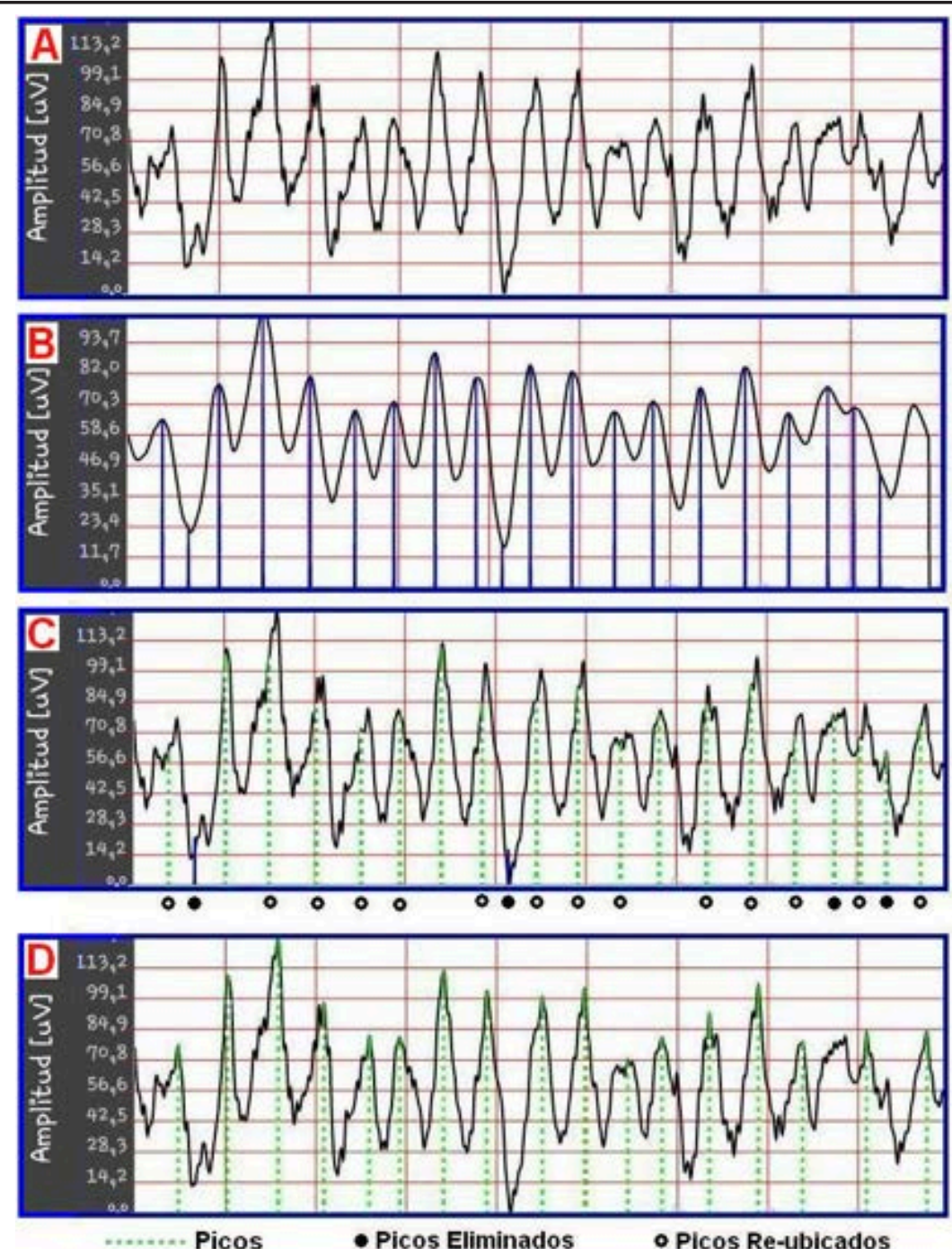

Fig. 8. Resultados Gráficos del algoritmo para la detección de Picos para un ritmo de FV
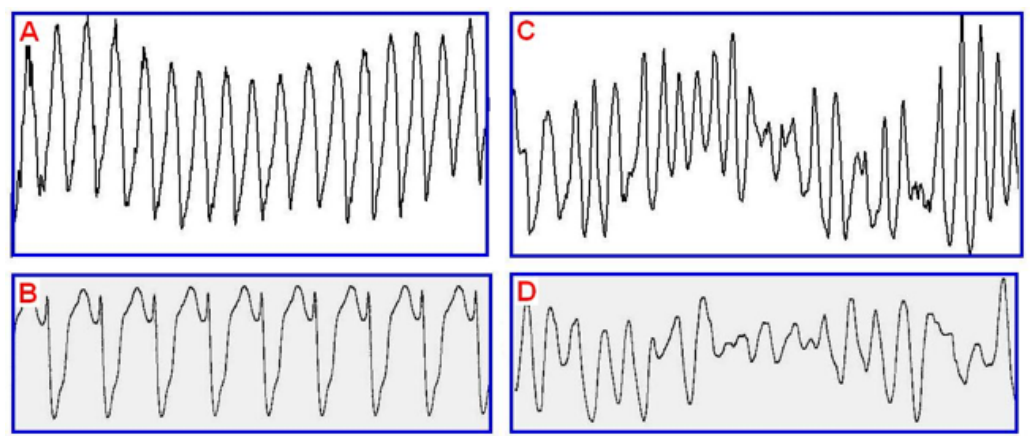

Fig. 9. Ejemplos de ritmos TV (izquierda) y FV (derecha)

\subsubsection{Detección de TVo FV}

La "Detección FV/TV" se basó en el parámetro "Dispersión de los Tramos RR" a partir de la detección de los picos usando en el algoritmo de Pan-Tompkins [16]. El algoritmo de Pan-Tompkins se concibió para la detección del $100 \%$ de los complejos QRS en ritmos ECG sanos $(R S)$. El algoritmo de Pan-Tompkins permite el análisis 
de características propias del complejo QRS como la pendiente, amplitud y ancho, se detectan los picos provocados por las contracciones ventriculares, logrando rendimientos muy buenos al ser implementado en ritmos sanos [17, 20, $14,18,2,23,12]$, pero su rendimiento baja considerablemente al momento de determinar la frecuencia cardiaca en arritmias de tipo ventricular como TV y FV. Existe una clara diferencia entre las contracciones de tipo ventricular en un ritmo sano $(R S)$ y un ritmo anormal $(T V, F V)$ en la Fig. 6 se aprecia esta diferencia.

Dada la diferencia entre picos sanos y no sanos, fue necesario realizar algunas modificaciones al algoritmo original de Pan-Tompkins para ajustarlo a la detección de picos provocados por las contracciones ventriculares presentes en las arritmias de interés. Estas modificaciones consistieron en la eliminación de los bloques de: "Derivación" y "Elevación al Cuadrado" del algoritmo original, y la adición de nuevos bloques que permitieron mejorar considerablemente la capacidad de detección de picos No Sanos. En la Fig. 7 se presenta el diagrama general para la detección de Picos No Sanos, el cual se compone de 5 partes, de las cuales la parte 2 es tomada del algoritmo de Pan-Tompkins y las restantes 1, 3, 4 y 5 son adaptaciones realizadas para mejorar la detección de picos anómalos de este algoritmo:

1. Conversión del Segmento a Valores Positivos: se busca que todos los datos del segmento evaluado sean valores positivos (array de valores positivos), pero a diferencia del algoritmo de Pan-Tompkins que hace esto mediante una etapa de "Elevación al Cuadrado" se optó por la detección del valor mínimo del segmento ECG que se está evaluando, dicho valor es sumado a todos los datos del segmento a manera de "offset" como se muestra en (3), de esta manera se evita enfatizar los grandes valores de amplitudes generadas durante una contracción ventricular al hacer uso de una transformación no lineal como una "elevación al cuadrado", pues conlleva a la atenuación de picos de poca amplitud que son importantes en las etapas posteriores.

$$
y(n)=y(n)+o f f s e t
$$

donde "offset = Mínimo valor del segmento evaluado"

2. Ventana Móvil de Integración: El algoritmo de PanTompkins [16] realiza este procedimiento para detectar los picos correspondientes a los complejos QRS; aquí se usa para enfatizar y suavizar los picos generados durante una contracción ventricular como los puntos de mayor pendiente, a través del cual se ubican los picos de forma aproximada. El cálculo se realiza mediante (4) usando un valor de $N$ de 40 muestras.

$$
y(n)=\left(\frac{1}{N}\right)[x(n-(N-1))+x(n-(N-2))+\ldots+x(n)]
$$

donde $N$ es el número de muestras dentro de la ventana de integración

3. Detección y Re-ubicación de Picos: Corresponde a determinar la ubicación aproximada (no definitiva) de los picos en base a los datos obtenidos de la ventana de integración. Posteriormente se hace una búsqueda hacia adelante y atrás en base a esta ubicación aproximada dentro del "arreglo de valores positivos" obtenidos del punto 1. Todo esto para obtener un arreglo con los picos que se cree son generados por las contracciones ventriculares con la ubicación definitiva de los mismos.

4. Eliminación de Picos que No superan el 50\% del valor del Pico Máximo: se procede con la eliminación de picos, para lo cual se identifica el pico de mayor amplitud de estos y se establece un umbral dinámico del $50 \%$ del pico máximo; eliminando los picos por debajo de este umbral.

5. Eliminación de Picos cuya distancia entre ellos es menor a 50 muestras: dado el proceso realizado en el punto 3, hay ciertos casos particulares en donde la reubicación de los picos genera que algunos picos queden reubicados muy cerca de otros, al punto que para una misma contracción ventricular se detecten dos picos lo cual es erróneo, con este paso se corrige este problema al eliminar el menor de los picos que estén a menos de 50 muestras de distancia entre ellos, con lo cual se define el array con los valores de los picos máximos definitivos, con este array se procede al cálculo de los Tramos RR.

En la Fig. 8 se presenta una señal ECG de una FV extraída de la base de datos del MIT para presentar la implementación de los 5 pasos anteriores para la detección de los picos. En imagen A se presenta el resultado de la "Conversión del Segmento a Valores Positivos (Paso 1)". En B se observa el resultado de la "Ventana Móvil de Integración (Paso 2)" aplicada a los datos obtenidos en el paso 1 , junto con los picos detectados preliminarmente. En C se presenta la "Detección y Re-ubicación de Picos (Paso 3)" que contiene todos los picos detectados (línea punteada), entre los que se encuentran los picos que serán eliminados $(\bullet)$ y los que serán reubicados (०) en el paso siguiente. Por último en $\mathrm{D}$, el resultado de la "Eliminación de Picos que No superan el 50\% del valor del Pico Máximo (paso 4)" y la "Eliminación de Picos cuya distancia entre ellos es menor a 50 muestras (paso 5)", lo que deja un total de 17 picos en comparación de los 20 calculados preliminarmente. La implementación de este algoritmo, como se observa en la imagen $\mathrm{D}$, es el cálculo preciso de los picos generados por las contracciones ventriculares, en este caso, para una FV. 
Con los valores de los picos máximos definitivos calculados mediante los 5 pasos anteriores, se procede a calcular la distancia entre picos consecutivos conocida como Tramos RR. En [9, 10, 12, 19, 23, 24] se ha usado el cálculo de los Tramos RR para discriminar entre arritmias regulares e irregulares. Mediante los Tramos RR, es posible establecer parámetros estadísticos, como la "Media" la cual es necesaria para el cálculo de la "Desviación Estándar" de los Tramos RR para usarlos como referencia al establecer si se trata de una TV o una FV; en un TV se espera que haya una dispersión menor ya que la ubicación de los picos suele ser consistente, más en el caso de una TV monomórfica la cual se caracteriza por tener picos de igual morfología (amplitud y distancia entre picos iguales) [1], mientras que la distancia entre tramos de una FV se caracteriza por tener una alta variabilidad debido a su naturaleza caótica y aleatoria [7]. La Fig. 9 se presenta dos ejemplos que arritmias tipo TV ( $A$, $B$ ), y dos arritmias tipo $\mathrm{FV}(C, D)$. Se puede observar que las TV presentan picos de amplitud similares al igual que las distancia entre ellos; en contraposición, los FV varían tanto en magnitud como en la distancia entre picos.

En la Fig. 12 se observa el diagrama de flujo del algoritmo desarrollado el cual está compuesto por 5 bloques principales correspondientes a: "1. Captura de la señal ECG”, "2. Pre-procesamiento", "3. Detección de Asistolia”, "4. Detección ritmo Sinusal" y por ultimo "5. Detección ritmo TV/FV". Los bloques 3, 4 y 5 es donde se realizan el procesamiento y posterior clasificación de los ritmos de interés, iniciando por la detección de Asistolia, si este no es el caso pasa a la siguiente detección ritmo Sinusal, si no corresponde a este ritmo se pasa al último bloque para determinar si corresponde a FV o TV. Cabe aclarar que una vez la trama evaluada es clasificada como alguno de estos ritmos el algoritmo se reinicia pasando a tomar otra trama de 5 seg para ser evaluada.

\section{Resultados y Discusión}

Tanto la aplicación de prueba para la validación del algoritmo, como la aplicación final (análisis de ritmo cardiaco en tiempo real), fueron desarrolladas en el entorno de desarrollo "Android SDK" y probadas en dispositivos móviles con el sistema operativo Android (una Tablet Samsung con Android 4.2.2, y tres Smartphone con Android 2.3.3, 4.1.2 y 4.4.2).
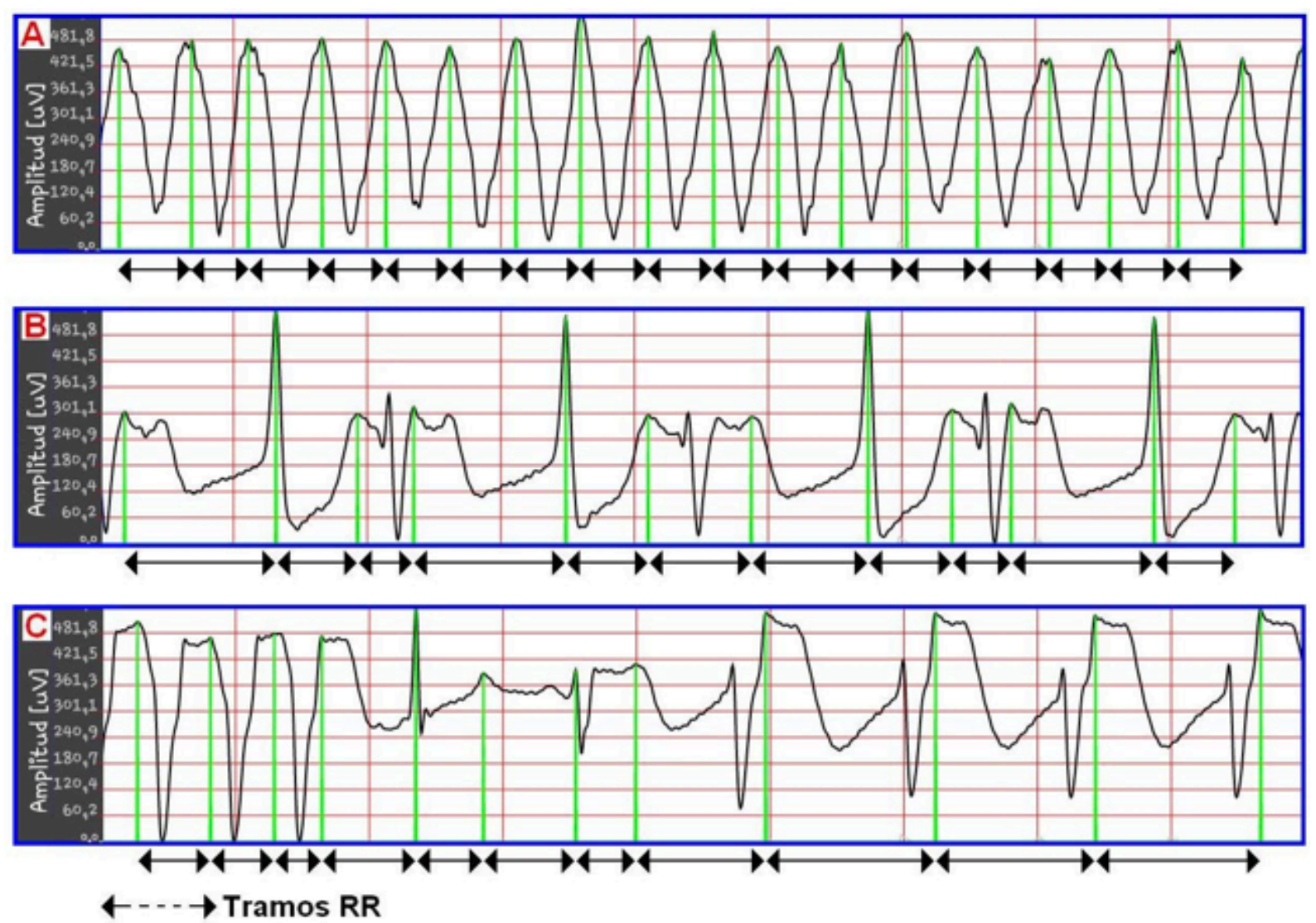

Fig. 10. Registros de TV monomórfica (A) y TV polimórfica (B, C). 


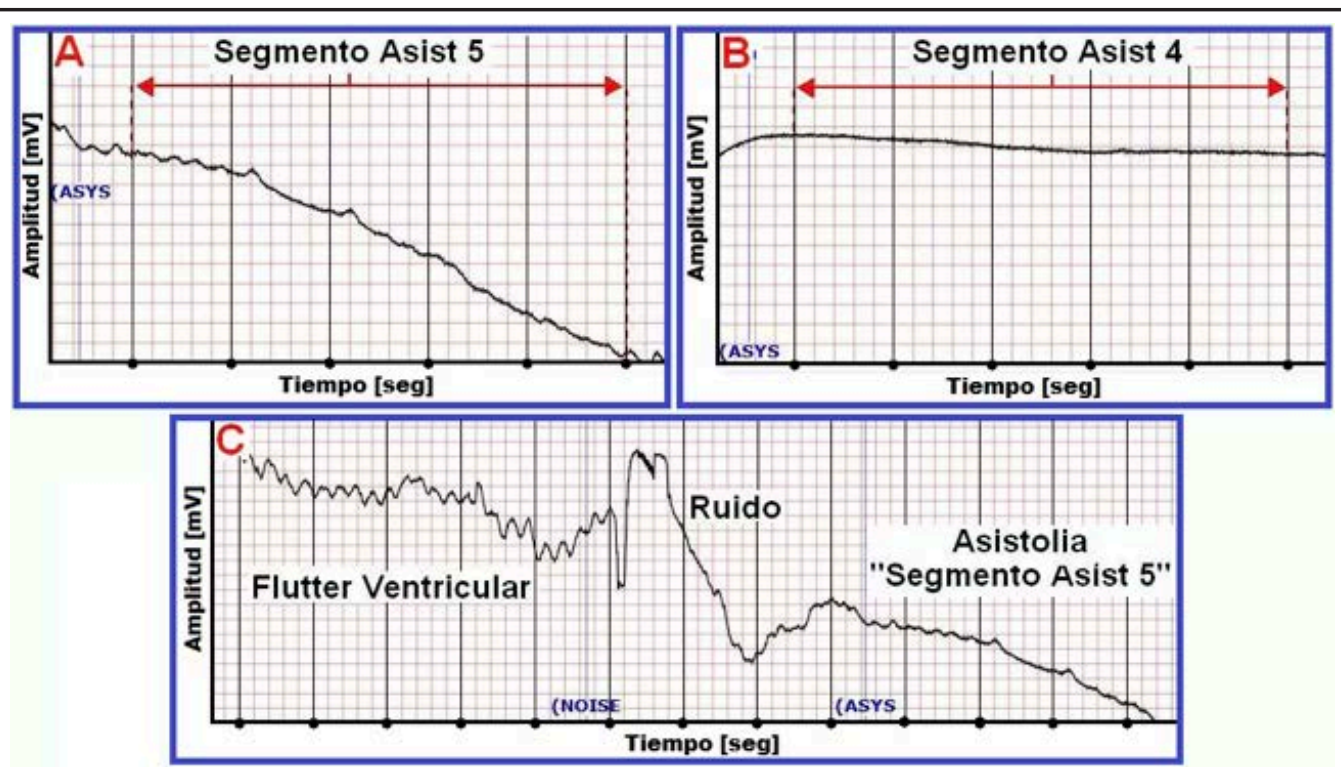

Fig. 11. Registros de Asistolia, A registro “Asist5" con variaciones, B registro "Asist4" sin variaciones y C transición entre arritmias

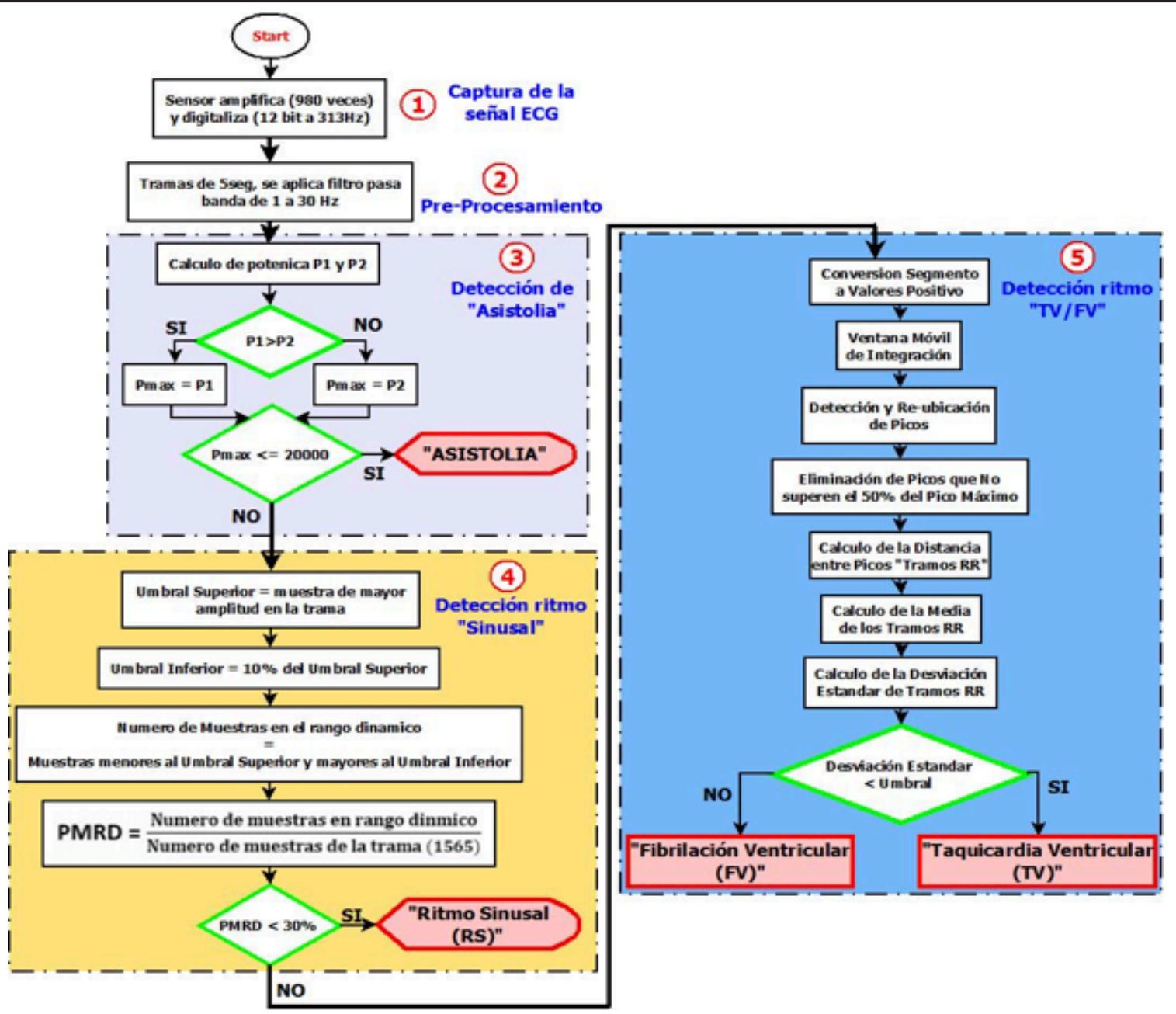

Fig. 12. Diagrama de flujo del algoritmo desarrollado, compuesto por 5 bloques principales 
La aplicación desarrollada permite la captura de la señal ECG en tiempo real y almacenarla en forma de archivo de texto (.txt). Dado que el registro se guarda con la fecha y hora en la memoria del dispositivo: el usuario puede llevar un registro de su actividad cardiaca durante la realización de tareas cotidianas. Las señales almacenadas pueden ser evaluadas por un especialista ya sea para identificar anomalías o determinar la efectividad de un tratamiento o medicamento para el control de un problema cardiaco. También tiene la capacidad de generar una alerta sonora en caso de detección de TV, FV o Asistolia, además de un indicador de nivel de batería.

En la Fig. 13 se presentan unas imágenes de la aplicación desarrollada ejecutándose en una Tablet Samsung con Android 4.2.2. A la izquierda la pantalla de visualización donde se observa de arriba hacia abajo; el registro ECG capturado por el sensor en tiempo real, la trama ECG ya filtrada que está bajo análisis la cual se actualiza cada $5 \mathrm{~s}$, un registro grafico de la frecuencia cardiaca del usuario, el nivel de carga de la batería del sensor, Seg-número de segundos transcurridos, M-número de muestras recibidas, FC-frecuencia cardiaca (lpm), botón para la activación y desactivación del análisis de la señal, el tipo de ritmo detectado que en este caso es NORMAL (RS), botón para conectarse y desconcertarse del sensor, casilla para ingresar el nombre del sensor al cual se quiere conectar, botón para grabar la señal ECG como archivo de texto, botón para pausar y reanudar la gráfica ECG vista en pantalla, casilla para ingresar el nombre del archivo de texto a guardar, casilla para ingresar el número de datos a guardar, botón para activar o desactivar el sonido (pulso cardiaco u alarma) y botón de salida de la aplicación. En la Fig. 13, a la derecha se observa la imagen del resultado final de la aplicación desarrollada usada por el usuario evaluando la señal ECG en tiempo real, donde se observan dos electrodos en el área del corazón, el electrodo de referencia en el lado inferior izquierdo del abdomen y el sensor al lado derecho de la cintura enviando los datos capturados a la Tablet de forma continua vía Bluetooth.

Para validar el sistema se usó la base de datos correspondientes al "MIT-BIH Normal Sinus Rhythm Database (nsrdb)", "MIT-BIH Malignant Ventricular Ectopy Database (vfdb)" y "MIT-BIH Arrhythmia Database (mitdb)". De los registros presentes en estas bases de datos los cuales tenían varios minutos de duración, se extrajeron los segmentos para 4 tipos de ritmos: RS, TV, FV y Asistolia, mediante el uso del toolbox para Matlab "WFDB", con el cual se elegían los segmentos de $5 \mathrm{~s}$ en diferentes tramos de los registros de la base de datos para descargarlos, posteriormente dichos segmentos eran re-muestreados dado que estas bases de datos tenían frecuencias de muestreo diferentes $(128 \mathrm{~Hz}, 250 \mathrm{~Hz} y$ $360 \mathrm{~Hz}$ respectivamente) a la del sensor $(313 \mathrm{~Hz})$. También se usaron 20 registros propios de señales RS normales, capturados con el sensor y la aplicación desarrollada, de tal forma que se pueda validar la parte del sistema relacionado con la captura y almacenamiento de señales ECG en el dispositivo móvil, bajo dos condiciones de captura: 10 registros de "RS Normal" obtenidos durante el reposo con frecuencias cardiacas entre los 60 a 130 lpm y otros 10 registros de "RS Rápidos" obtenidos durante la actividad física con frecuencias cardiacas mayores a $130 \mathrm{lpm}$ y memores a $190 \mathrm{lpm}$.

En la Tabla 1 se presentan el número de registros ECG usados para la validación del algoritmo por tipo de ritmo y origen. La Tabla 2 se presenta la matriz de confusión en la clasificación del ritmo cardiaco RS, TV, FV y Asistolia. Para el análisis de la tabla, es importante conocer la evolución de una falla cardiaca fatal, la cual inicia con una Taquicardia Ventricular $(T V)$, continúa con una Fibrilación Ventricular $(F V)$ y termina con la ausencia total de actividad cardiaca (Asistolia) en la mayoría de los casos.

Tabla 1. Tipo, Origen y Número de registros para la validación del algoritmo

\begin{tabular}{|c|c|c|}
\hline Tipo de Ritmo & Origen & Número de registros usados \\
\hline RS Normal & MIT-BIH & 20 \\
\hline TV & MIT-BIH & 20 \\
\hline $\mathbf{F V}$ & MIT-BIH & 20 \\
\hline Asistolia & MIT-BIH & 10 \\
\hline RS Normal & Propio & 10 \\
\hline RS Rápidos & Propio & 10 \\
\hline \multicolumn{2}{|c|}{ Total } & 90 \\
\hline
\end{tabular}

Tabla 2. Matriz de Confusión Resultados Totales

\begin{tabular}{|c|c|c|c|c|c|c|}
\hline & \multirow{3}{*}{ Tipo de Ritmo } & \multicolumn{5}{|c|}{ Medido } \\
\hline & & \multirow{2}{*}{$\begin{array}{c}\text { Ritmo No Letal } \\
\text { RS } \\
\end{array}$} & \multicolumn{3}{|c|}{ Ritmos Letales } & \multirow{2}{*}{ Total } \\
\hline & & & TV & FV & Asistolia & \\
\hline \multirow{5}{*}{ Real } & RS & $\begin{array}{c}40 \\
(100 \%) \\
\end{array}$ & & & & 40 \\
\hline & TV & & $\begin{array}{c}11 \\
(55 \%) \\
\end{array}$ & $\begin{array}{c}9 \\
(45 \%) \\
\end{array}$ & & 20 \\
\hline & FV & & $\begin{array}{c}5 \\
(25 \%)\end{array}$ & $\begin{array}{c}15 \\
(75 \%)\end{array}$ & & 20 \\
\hline & Asistolia & & & $\begin{array}{c}1 \\
(10 \%)\end{array}$ & $\begin{array}{c}9 \\
(90 \%)\end{array}$ & 10 \\
\hline & Total & --- & --- & --- & --- & 90 \\
\hline
\end{tabular}


Como se observa en la tabla, en el caso de los registros de RS se obtuvo una clasificación correcta del $100 \%$, independientemente de la frecuencia cardiaca (RS Normal y RS Rápidos), lo cual significa una excelente discriminación entre Ritmos Normales $(R S)$ y Ritmos Letales (TV, FV y Asistolia).

Una parte muy importante del sistema, es la detección de los picos previo a la clasificación del ritmo como TV o FV. Así, en los resultados de la detección de picos durante una TV se logró una detección del $100 \%$ de los picos, mientras que para las FV se logró una detección del $83 \%$. La no detección de algunos picos se debe a que al no cumplir criterios como la amplitud o distancia mínima entre ellos, son descartados y considerados como ruido. Dado que en 3 de los 5 segmentos de FV que fueron clasificados erróneamente como TV (Tabla 2) el porcentaje de detección de picos era del $100 \%$, se puede concluir que los casos de mala clasificación están más relacionados con la regularidad de los Tramos RR entre picos.
El bajo porcentaje de identificación de los ritmos de TV (55\%), están relacionados con el hecho que los registros usados de TV, no sólo contienen señales TV monomórficas [9] sino que contienen señales TV polimórficas. En el primer caso, las señales se caracterizan por tener un patrón regular y claro entre sus picos que facilitan su reconocimiento; mientras que las TV polimórficas [9] son muy irregulares y su forma de onda tiende a asemejarse al de una FV. En la Fig. 10 se presentan tres registros de TV, en A se observa una TV monomorfica (regular) clasificada correctamente la cual presenta pocas variaciones en las longitudes de los Tramos RR, mientras que en $\mathrm{B}$ y $\mathrm{C}$ se observan registros de TV polimórficos que por lo general conllevan a que el parámetro "Dispersión de Tramos RR" presente valores cercanos a los presentes en una FV. Por lo anterior, todos los casos bien clasificados de TV fueron señales monomorficas, mientras que los 9 registros con características de señales TV polimórficas fueron erróneamente clasificados como FV.
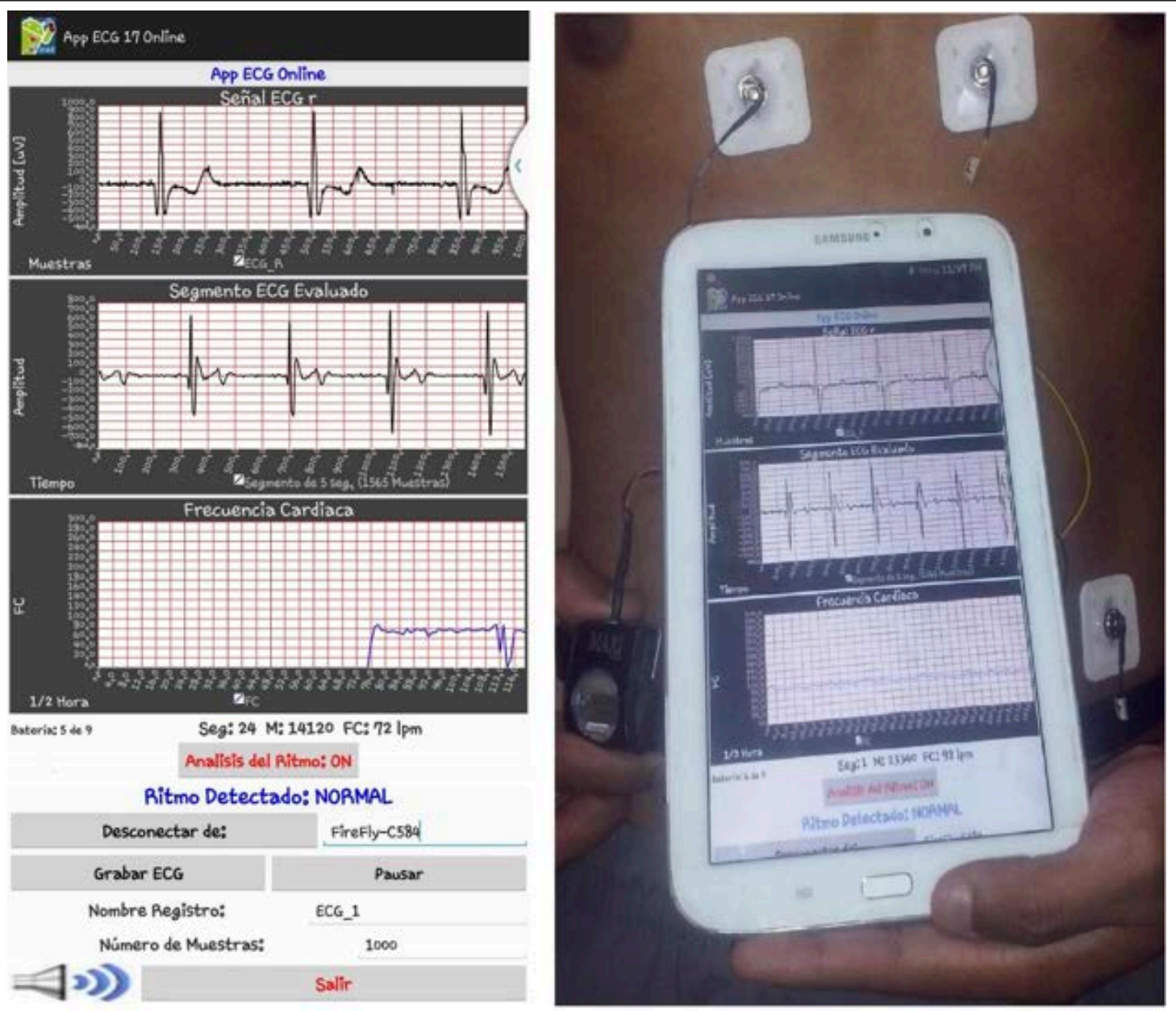

Fig. 13. Sistema en funcionamiento, izquierda captura de pantalla de la aplicación ejecutándose en Tablet, derecha sistema usa-do por el usuario ejecutando en tiempo real 
El porcentaje de reconocimiento de los ritmos de FV fue de $75 \%$. Las fallas en su detección, en forma similar al caso anterior, se producen por pocas variaciones en el valor de los Tramos RR, en donde los picos son más regulares que irregulares, y por tanto, el parámetro "Dispersión de Tramos RR" presentó valores pequeños cercanos a los relacionados con las señales TV.

En cuanto a los ritmos de Asistolia se logró una clasificación correcta del $90 \%$, y las señales restantes fueron clasificadas erróneamente como una FV. En la Fig. 11 se observan dos registros de Asistolia, la gráfica A corresponde al registro "Asist5" clasificado como FV, ya que presenta ciertas variaciones (pequeños picos) que sobrepasan el umbral definido para que sea considerada como Asistolia. En B se observa el registro de Asistolia "Asist 4 " sin variaciones de algún tipo que facilitara la correcta clasificación. En $\mathrm{C}$ se observa un fragmento (registro MIT, vfdb/424, 00:25:29 a 00:25:42) que contiene secuencialmente un Flutter Ventricular $(F V L)$, Ruido y una Asistolia (registro "Asist5"). Como se produce una sucesión de ritmos cardiacos, es bastante que la transición entre ellos sea susceptible de error, como en este caso, que el Flutter Ventricular cambia a una Asistolia. Es importante destacar que una detección errónea de un segmento puede ser corregido $5 \mathrm{~s}$ después, durante el análisis del siguiente segmento ECG.

\section{Conclusiones}

En este artículo se presenta un sistema inalámbrico, portable y menos invasivo que permite el registro y monitoreo de señales ECG en tiempo real. Los algoritmos usados tienen un bajo costo computacional para garantizar el análisis de las señales en tiempo real, pues la aplicación desarrollada pueda ejecutarse simultáneamente con aplicaciones de navegación, reproducción multimedia, llamadas, etc.

El sistema es capaz de discriminar el 100\% de las veces entre Ritmos No Letales (RS) y Ritmos Letales (TV, FV y Asistolia). Los errores de identificación del sistema se produjeron entre anomalías cardiacas consecutivas en la evolución de arritmias cardiacas. Mientras que las señales TV monomorficas son correctamente identificadas, la mayoría de las TV polimórficas se identifican como FV. Del mismo modo, las señales FV con son confundidas con una TV, probablemente durante la transición de TV a FV. Finalmente las señales $\mathrm{FV}$ pueden confundirse con Asistolia, la etapa final en la evolución de anomalías cardiacas.

En caso de errores, el sistema es bastante sensible a recuperarse durante el análisis de la trama subsecuente, 5 $\mathrm{s}$ después; por lo que el efecto en la rápida atención de las anomalías es menor. La aplicación permite la captura de la señal ECG en tiempo real y almacenarla en forma de archi- vo de texto (.txt). Ya que se puede guardar con la fecha y hora en la memoria del dispositivo: el usuario puede llevar un registro de su actividad cardiaca durante la realización de tareas cotidianas. Las señales almacenadas pueden ser evaluadas por un especialista ya sea para identificar anomalías o determinar la efectividad de un tratamiento o medicamento para el control de un problema cardiaco.

Aunque existen otros sistemas más precisos, por lo general implican la adquisición de sensores especiales que incrementan los costos del sistema, mientras que el trabajo propuesto busca aprovechar los teléfonos inteligentes con los que el usuario ya cuenta, y sólo requiere el uso de electrodos de bajo costo. Así mismo, la aplicación identifica automáticamente las anomalías para disminuir el tiempo de atención de sus usuarios en caso de una anomalía cardiaca, en contraposición muchos de los sistemas encontrados se limitan a registrar la señal y almacenarla para que sea analizada por un especialista posteriormente.

\section{Trabajos Futuros}

En caso de detectar alguna anomalía cardiaca se genera una alarma sonora; sin embargo, se puede explotar la capacidad de conectividad que poseen los dispositivos móviles actuales para enviar mensajes de alerta a familiares $\mathrm{u}$ hospitales cercanos, ya sea por internet o SMS. Así mismo, mediante el módulo GPS integrado en estos dispositivos se pueden enviar la ubicación del paciente.

Es posible agregar otros parámetros biomédicos como temperatura y nivel de oxígeno en la sangre para obtener un sistema más integral en cuanto al monitoreo de pacientes de forma remota, no solo con problemas cardiacos, sino también con otro tipo de patologías como la diabetes, la hipertensión, etc.

Con la integración de sensores para la captura de bioseñales con dispositivos móviles de uso cotidiano como los PDA, se puede obtener una herramienta muy versátil para el monitoreo de cualquier padecimiento que demande una constante supervisión, reduciendo el tiempo de detección y respuesta ante cualquier emergencia médica, de tal forma que aumenten las probabilidades de supervivencia del paciente, con el uso de tecnología como Smartphones o Tablets con la que un gran número de personas ya cuentan.

\section{REFERENCIAS}

[Web 1] Organización Mundial de la Salud, Enfermedades Cardiovasculares (Enero 2015), consultado Marzo 2015, disponible en: http://www.who.int/mediacentre/factsheets/fs317/es/

[Web 2] El Espectador, "Estas son las principales causas de muerte en Colombia", consultado Octubre 2018, disponible en: https://www. elespectador.com/noticias/nacional/estas-son-las-principalescausas-de-muerte-en-colombia-articulo-730249 
[Web 3] Wikipedia, Monitor Holter (2017), consultado Octubre 2018, disponible en http://es.wikipedia.org/wiki/Holter

[Web 4] AliveCor. Mobile ECG. Consultado Diciembre 2013 en: http:// www.alivecor.com/

[Web 5] Wikipedia. Desfibrilador Automático. Consultado Febrero $2015 \quad$ en: http://es.wikipedia.org/wiki/ Desfibrilador_autom\%C3\%A1tico_implantable.

[Web 6] Mio Alpha. Heart Rate Sport Watch. Consultado Diciembre 2013 en: http://www.mioglobal.com/EN-US/Mio-ALPHA-2Heart-Rate-Sport-Watch/Product.aspx

[Web 7] Beurer. Pulsómetros de Beurer. Consultado Diciembre 2013 en: https:/www.beurer.com/web/es/products/heart_rate_monitors/ heart rate monitors.php

[Web 8] Sport Pulse Ring. Consultado Diciembre 2013 en: http://www. vikingopticalcentres.co.uk/media/pulsring-manual-english.pdf

[1]. Libro: "El Electrocardiograma su interpretación practica" (Christian W. Hamm, Stephan Willems, $3^{\mathrm{a}}$ edición página 146).

[2]. Articulo: Alvaro J. Saldarriaga, Jairo J. Pérez, J. Restrepo, J. Bustamante, "A mobile application for ambulatory electrocardiographic monitoring in clinical and domestic environments", Grupo de Dinámica Cardiovascular, Universidad Pontificia Bolivariana, Medellín, Colombia, 2013.

[3]. Artículo: Juan Pablo Tello P., Oscar Manjarres, Mauricio Quijano, Arcelio Ulises Blanco, "Remote Monitoring System of ECG and Temperature Signals using Bluetooth", 2012 International Symposium on Information Technology in Medicine and Education, Universidad del Norte, Barranquilla, Colombia, 2012.

[4]. Artículo: Issac. R, Ajaynath M.S., "CUEDETA: A Real Time Heart Monitoring System Using Android Smartphone", Dept. of Computer Science \& Engineering College of Engineering, Kallooppara Kerala, India, 2012.

[5]. Tesis: Cristhoffer Sebastián Novoa Tiznado, "Monitoreo de Signos Vitales para el Cuidado de Adultos Mayores Usando Smartphones", Tesis de grado, Ingeniero Civil en Telecomunicaciones, Chile, 2012.

[6]. Artículo: Jorge Alaniz, José Manuel Valencia, Cristián Castillo, Bereniz Castañeda, Arturo Serrrano, "Monitoreo de Frecuencia Cardiaca a través de Dispositivos Móviles", Universidad Autónoma de Baja California, Centro de Investigación Científica y Educación Superior de Ensenada, México - 2012.

[7]. Tesis: Urtzi Ayesta Morate, "Clasificación de arritmias cardiacas mediante el algoritmo PMRD-C(n)", Tesis de grado, Ingeniero de Telecomunicación, Pamplona, 2001.

[8]. Artículo: Mohd Afzan Othman, Norlaili Mat Safri, Ismawati Abdul Ghani, Fauzan Khairi Che Harun, "Characterization of Ventricular Tachycardia and Fibrillation Using Semantic Mining", Revista Computer and Information Science, Vol. 5, No. 5; 2012. Malaysia, 2012.

[9]. Artículo: José M. Ormaetxe-Merodio, Jesús D. Martínez-Alday, María F. Arcocha-Torres y Rubén García-Martín, "Programación del desfibrilador automático implantable", Laboratorio de Electrofisiología. Servicio de Cardiología. Hospital de Basurto. Rev Esp Cardiol Supl. 2008; 8: 65A-75A, Bilbao, España.

[10]. Artículo: Pankaj K. Gakare, Abhilasha M. Patel, Jignesh R. Vaghela, R. N. Awale, "Real Time Feature Extraction of ECG Signal on Android Platform", 2012 International Conference on Communication, Information \& Computing Technology (ICCICT), Oct. 19-20, Mumbai, India.
[11]. Tesis: Unai Irusta Zarandona, "New Signal Processing Algorithms for Automated External Defibrillators", Tesis de Doctorado, Doctor of Science, Bilbao, 2010.

[12]. Artículo: H. M. K. G. S. Jayasumana, T. M. U. A. S. Thennakoon, C. M. R. B. Chandrasekara, M. T. Sandaruwan, A. A. Pasqual and N. D. Nanayakkara, "A Stand-alone ECG Abnormality Detector", University of Moratuwa, Katubedda, Sri Lanka, 2010.

[13]. Artículo: Víctor Castro Urda, Jorge Toquero Ramos, Pablo Aguiar Souto, Pablo Pavía, Jesús Mirelis e Ignacio Fernández Lozano, "Criterio de detección por morfología en el desfibrilador automático implantable", Madrid-España, 2007.

[14]. Artículo: Anton Amann, Robert Tratnig, Karl Unterkofler, "Reliability of old and new ventricular fibrillation detection algorithms for automated external defibrillators", 2005.

[15]. Artículo: A. R. Fernández Fornés, "Validación de un Conjunto de Algoritmos para la Detección de Fibrilación Ventricular. Resultados Experimentales", Memorias V Congreso de la Sociedad Cubana de Bioingeniería, Habana 2003, Junio 10 al 13 de 2003 .

[16]. Artículo: Jiapu Pan, Willis j. Tompkins, "A Real-Time QRS Detection Algorithm", IEEE Transactions on Biomedical Engineering, vol. bme-32, no. 3, march 1985.

[17]. Artículo: Edwin Andrés Quintero Salazar, Juan David Valencia Parra, "Simulación del algoritmo Pan-Tompkins para la detección de intervalos QRS en tiempo real", Revista Memorias, vol. 10, núm. 18, pp. 73-81, Abril 2012.

[18]. Artículo: Shijie Zhou, Zichen Zhang, Jason Gu, Boston), "TimeDomain ECG Signal Analysis Based on Smart-Phone", 33rd Annual International Conference of the IEEE EMBS Boston, Massachusetts USA, August 30 - September 3, 2011.

[19]. Tesis: Mauricio Enrique Reina Fiore, "Diseño y Análisis de Confiabilidad de un Algoritmo para la Detección de Fibrilación Auricular", Tesis de Maestría, Ingeniería Biomédica, Universidad Simón Bolívar, Febrero 2006.

[20]. Tesis: Mauricio Orozco Alzate, "Clasificación de Arritmias Cardíacas usando Transformada Wavelet y Técnicas de Reconocimiento de Patrones", Tesis de grado, Ingeniero Electrónico, Universidad Nacional, Manizales-Colombia, 2003.

[21]. Tesis: Alejandro Rodríguez Ibáñez, "Análisis y Desarrollo de Métodos para la Detección de Apneas en Electrocardiograma", Tesis de grado, Ingeniería Informática, Universidad Autónoma de Barcelona, 13 de Septiembre de 2013.

[22]. Artículo: Fei Zhang, Pengye Li, Fan Jiang and Dakun Lai, "A Shockable Rhythm Detection Algorithm for Automatic External Defibrillators by Combining a Slope Variability Analyzer with a Band-Pass Digital Filter", 2014 IEEE Workshop on Electronics, Computer and Applications, Chengdu, China -2014.

[23]. Artículo: Joseph J. Oresko, Zhanpeng Jin, Jun Cheng, Shimeng Huang, Yuwen Sun, Heather Duschl, and Allen C. Cheng, "A Wearable Smartphone-Based Platform for Real-Time Cardiovascular Disease Detection Via Electrocardiogram Processing", IEEE Transactions on Information Technology in Biomedicine, vol. 14, NO. 3, may 2010.

[24]. Tesis: Javier Enrique Gonzalez B., "Procesamiento digital de señales electrocardiográfica con tecnología DSP orientado al análisis de variabilidad de la frecuencia cardiaca", Trabajo de investigación, Santander, Colombia, 2005.análisis de variabilidad de la frecuencia cardiaca", Trabajo de investigación, Santander, Colombia, 2005. 